\title{
Primary sternal osteomyelitis
}

\author{
CA KELLY, MN CHETTY
}

From the Regional Cardiothoracic Centre and the Medical Unit, Freman Hospital, Newcastle upon Tyne

Primary osteomyelitis of the sternum is a rare condition. Infection may follow chest trauma and is a recognised complication of open heart surgery, with a reported incidence of $1-2 \% .^{\prime}$ Since the detailed review by Wilensky and Samuels in $1926^{2}$ there have been only occasional reports. We describe a case of chronic sternal infection without preceding trauma and present a review of published reports.

\section{Case report}

A 40 year old housewife presented with pain and swelling in the front of her chest five months after an acute self limiting diarrhoeal illness in Tenerife. The swelling had developed over five days and was associated with weight loss of $6.3 \mathrm{~kg}$. Examination revealed an inflammed tender area over the manubrium measuring $3 \times 2 \mathrm{~cm}$ and pyrexia of $37.4^{\circ} \mathrm{C}$. There was no lymphadenopathy and no other focus of infection was found. Radiographs showed widening of the sternum without lysis. The white cell count was $11.3 \times 10^{9} / 1$; the initial erythrocyte sedimentation rate was 65 Westergren units in one hour, rising to 115 over the next week. Needle aspiration of the sternum showed scanty Gram negative micrococci, considered to be contaminants. Blood, stool, urine, and marrow cultures were negative and serological examination for brucella and typhoid organisms also gave negative results. Culture for acid fast bacilli was negative. A needle biopsy of the sternum was performed and histological examination showed features of chronic non-specific inflammation with reactive woven bone formation, but no organisms were seen. A gallium bone scan (figure), which has been of diagnostic value in previous cases, ${ }^{3}$ showed increased uptake along the sternum suggestive of osteomyelitis and treatment was started with fusidic acid on the assumption that Staphlococcus aureus was the most likely pathogen. There was no improvement in her condition after one month so treatment was discontinued and surgical exploration of the sternum was undertaken. Material obtained showed microscopic features of chronic inflammation. The curettings obtained did not contain pus and again no organisms were identified.

Doxycycline was prescribed. Symptomatic relief was gradually achieved, the signs resolved, and the patient remains well several months later.

\footnotetext{
Address for reprint requests: Dr CA Kelly, Regional Cardiothoracic Centre, Freeman Hospital, Newcastle upon Tyne NE7 7DN.
}

Accepted 25 March 1985

\section{Discussion}

We suggest that this case illustrates some unusual features of a rare disease. The pathogen has rarely been difficult to isolate and the condition is unusual in females over 30 years old. Although no causative organism was proved in the case described, the circumstantial evidence of chronic infection is compelling. A Gram negative coccus was implicated as a possible infective agent in one other report ${ }^{4}$; the patient affected responded rapidly to tetracycline. Most patients respond promptly to appropriate treatment and the persistence of symptoms beyond two months is an unusual feature.

The age at diagnosis ranges from 3 months to 41 years, only eight cases having occurred in children. ${ }^{24}$ This pattern has not changed since antibiotics became widely used but the incidence of the condition has fallen sharply since the 1940 s. Mortality has also been radically altered by the use of antibiotics. Almost half of the 24 patients reported up to 1926 had a fatal outcome to their illness, while there has been only one reported death in the subsequent 27 patients.

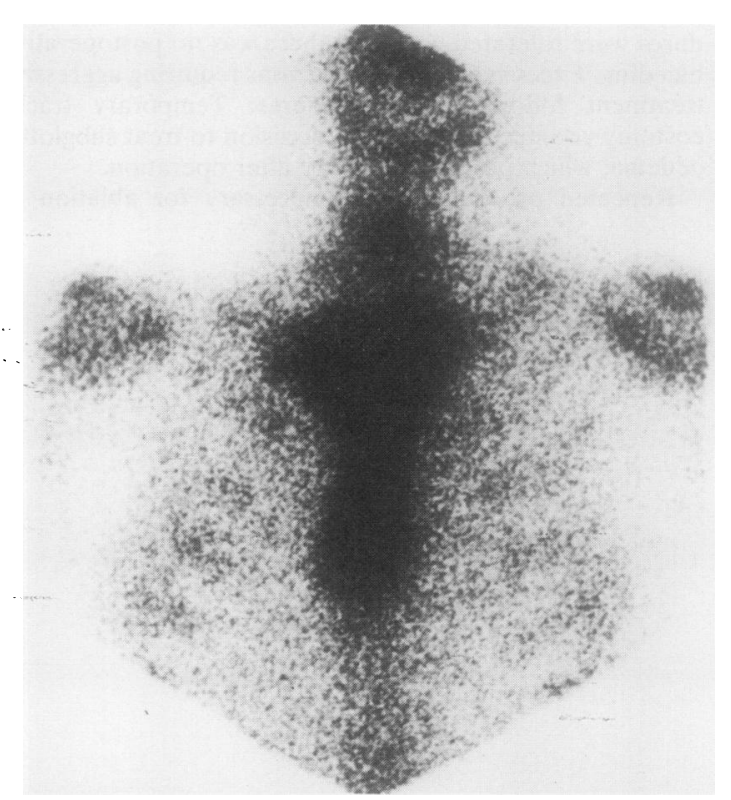

Gallium bone scan showing an area of increased uptake over the sternum. 
Organisms isolated before and after 1926 in primary sternal osteomyelitis

\begin{tabular}{lll}
\hline & Up to 1926 & After 1926 \\
\hline Staphlococcus aureus & $3(3)^{*}$ & $10(6)^{*}$ \\
Pseudomonas aeruginosa & 0 & $7 \dagger$ \\
Mycobacterium tuberculosis & 0 & 6 \\
Salmonella typhi & 2 & 0 \\
Micrococcus & 0 & 2 \\
Haemolytic streptococcus & 0 & 1 \\
Nothing confirmed & 19 & 1 \\
Total & 24 & 27 \\
\hline
\end{tabular}

*Numbers in parentheses indicate cases occurring after trauma.

†Including six heroin addicts.

The number of infections occurring after trauma to the chest wall may be of interest. A history of trauma was obtained in only 11 cases and eight of these have occurred since 1926. Possibly some of the earlier cases followed unrecorded trauma, as the information available is often incomplete. All but two post-traumatic infections have been caused by staphlococci; two of these have followed bone marrow aspiration and one has occurred after cardiac resuscitation. ${ }^{6}$ The two exceptions both occurred in heroin addicts, in whom Pseudomonas aeruginosa was the pathogen.?

The infective agent is sometimes predictable. Staphlococcus aureus is the commonest, accounting for $47 \%$ of all cases where positive bacterial identification was achieved (table). Recently seven cases of pseudomonas osteomyelitis have been reported from North America. ${ }^{78}$ Six of the patients were established heroin addicts. All required debridement and curettage of the sternum in addition to antibiotics before healing was achieved. There have also been some reports of sternoarticular septic arthritis caused by Pseudomonas. " These patients have invariably been heroin addicts.

In the preantibiotic era identification of the responsible organism was frequently not achieved. In only $18 \%$ of the 24 cases reported by Wilensky and Samuels were bacteria identified. ${ }^{2}$ Three were due to Staphlococcus aureus and two caused by Salmonella sp. Although many others may have followed a staphlococcal septicaemia, from the history available several were probably tuberculous. Brown and Trenton ${ }^{10}$ in 1951 reviewed 10 cases of chest wall sepsis, of which four had the sternum affected. All were tuberculous in origin and required resection of the affected bone and sometimes of underlying tissues. Two further cases of tuberculous osteomyelitis of the sternum have since been described." Perhaps surprisingly, only one case has been attributed to Streptococcus pneumoniae. ${ }^{12}$ This -was part of a fulminant systemic infection and the patient died despite treatment with penicillin.

Sternal infection after sternotomy is now much more common than primary infection, but the organisms responsible may differ. Secondary infection has been caused by Candida sp.' Staphlococcus albus has also been implicated but Staphlococcus aureus remains the commonest cause of osteomyelitis in this group.

Treatment has been recently reviewed. ${ }^{78}$ Before antibiotics became available, surgical exploration and resection offered the only definitive treatment. Antibiotics greatly reduced the need for surgery but more recently the benefit of a limited resection and curettage has become apparent. These are particularly useful in pseudomonal and tuberculous infections and may also be of benefit in chronic disease where antibiotics have failed to produce a cure.

Our thanks are due to Dr S Nariman for permission to report this case.

\section{References}

1 Culliford AT, Cunningham JN, Zeff RH, Isom OW, Teiko P, Spencer FC. Sternal and costochondral infection following open heart surgery.J Thorac Cardiovasc Surg 1976;72:714-26.

2 Wilensky AO, Samuels SS. Osteomyelitis of sternum. Ann Surg 1926;83:206-16.

3 Smith PW, Petersen RJ, Feruc RM. Gallium scan in sternal osteomyelitis. Am J Radiol 1979;132:840-1.

4 Biesecker GL, Aaron BL, Mullen J. Primary sternal osteomyelitis. Chest 1973;63:236-8.

5 Emanuel B, Young N. Recurrent osteomyelitis of the sternum. Ill Med J 1977;152:110-2.

6 Enat R, Pollack S, Wiener M, Barzilai P. Osteomyelitis in fractured sternum after cardio-pulmonary resuscitation. $N$ Engl $J$ Med 1979;301:108-9.

7 Mandal AK, Fiala M, Oparah SS, Thadepalli H. Osteolytic lesion indicating pseudomonas sternal osteomyelitis. Arch Surg 1978;111:776-8.

8 Mirsepasi MH, Gazzaniga AB, Bartlett RH. Surgical treatment of primary osteomyelitis. Ann Thorac Surg 1975;19:698-703.

9 Goldin RH, Chow AW, Edwards JE, Louie JS, Guzel B. Sternoarticular septic arthritis in heroin users. $N$ Engl J Med 1973;289:616-7.

10 Brown RB, Trenton J. Chronic abscesses and sinuses of the chest wall. Ann Surg 1952;135:44-51.

11 Richter R. Isolated tuberculosis of the sternum [German]. RORFO 1983;139:132-5.

12 Maier HC. Infections of costal cartilages and sternum. Surg Gynaec Obstet 1947;84:1038-44. 